\section{Glancing-angle diffraction anomalous fine structure of InAs quantum dots and quantum wires}

\author{
S. Grenier ${ }^{a}$, M. G. Proietti ${ }^{a, b}$, H. Renevier ${ }^{a}$, \\ L. Gonzalez ${ }^{c}$, J. M. García ${ }^{c}$, J. M. Gérard ${ }^{d}$, and \\ J. García
}

a'Laboratoire de Cristallographie, C.N.R.S., B.P. 166, 38042 Grenoble Cedex 09, France.

${ }^{b}$ Instituto de Ciencia de Materiales de Aragón, C.S.I.C.Universidad de Zaragoza, c. Pedro Cerbuna 12, 50009

Zaragoza, Spain

'Instituto de Microelectrónica de Madrid, C.S.I.C., c. Isaac

Newton 8, 28760 Tres Cantos, Spain

${ }^{d}$ FranceTelecom, C.N.E.T.- P.A.B., Laboratoire de Bagneux, 92220 Bagneux, France.

Email:proietti@posta.unizar.es

We have performed Diffraction Anomalous Fine Structure measurements at the As K-edge of self-growth $\operatorname{InAs} / \operatorname{InP}(001)$ Quantum Wires and InAs/GaAs(001) Quantum Dots. The samples have been grown by Molecular Beam Epitaxy and their equivalent thickness is of 2.5 monolayers. We have measured the (440) and (420) Bragg reflections in glancing-angle scattering geometry, at incidence angles close to the substrate critical angle. We demonstrate the feasibility of the experiment reporting, for the first time, Diffraction Anomalous Fine Structure spectra of such low coverage epitaxial layers, and we show that the analysis of the Diffraction Anomalous Fine Structure lineshape together with the analysis of oscillatory part of the signal, can provide information about composition and strain of the nanostructures.

Keywords: DAFS, nanostructures, Quantum Dots, Quantum Wires, anomalous diffraction.

\section{Introduction}

Patterning often occurs in nature through self-organization. A "spontaneous" self-ordering process can be used to obtain periodically structured materials at a nanometer scale. In the case of semiconductors, ordered arrays of crystalline dots and wires (QDs and QWrs) can be obtained during the first steps of epitaxial growth. The dimension of these self-assembled structures are in the nanometer range and they can provide a series of attractive quantum properties to fabricate the last and next generation of optoelectronic devices (Xie, 1995).

The QDs auto-organization is driven by the strain at the interface due to the lattice mismatch between substrate and epilayer. The quantum arrays are characterized mainly in terms of optical (luminescence, photoreflectance) and vibrational properties (Raman), while AFM and TEM give an image of the shape and an idea of the homogeneity of the islands. The nanostructure composition is not well known, since reactions with the substrate atoms or segregation mechanisms of the impinging species can occur.

Diffraction Anomalous Fine Structure (DAFS) offers the unique advantage of joining the chemical selectivity of XAFS with the spatial and site selectivity of X-Ray Diffraction, allowing a closer look to the local structure of these nanoobjects.

It allows to recover information regarding the atoms that belong to the ordered core of the nanostructure. For example we show in the following that the As atoms belonging to the oxide «dead » layer on the sample surface, which strongly affect EXAFS, are transparent for DAFS. Moreover, if the crystallographic structure of the system is known the analysis of the DAFS lineshape can also give the sample composition. It reveals in our case, an appreciable concentration of $\mathrm{P}$ and $\mathrm{Ga}$ in the InAs epilayer of QWrs and QDs respectively.
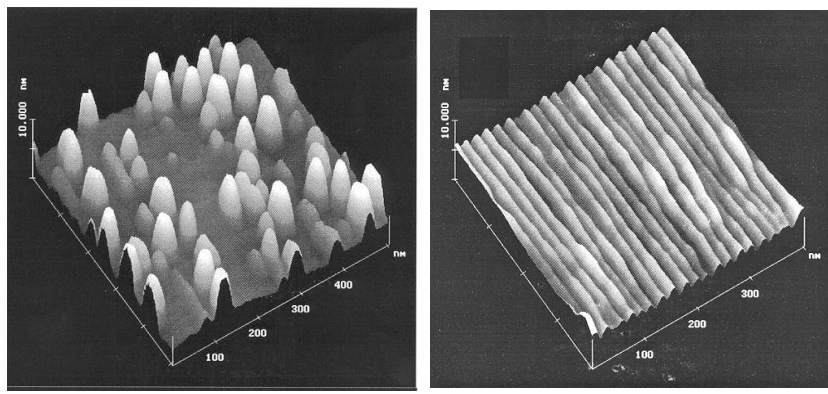

Figure 1. AFM tridimensional view of InAs QDs and QWrs on InP buffer (Gonzalez, 2000).

\section{Experimental}

Samples have been grown by Molecular Beam Epitaxy onto $\mathrm{InP}(001)$ (Fig. 1) and $\mathrm{GaAs}(001)$ wafers, after having grown a thick InP and GaAs buffer layer respectively. The equivalent InAs coverage is of about 2.5 Mono Layers (ML). The height of the dots is of about $7 \mathrm{~nm}$, their average diameter is of $23 \mathrm{~nm}$, and the density is of $2 \times 10^{10} \mathrm{~cm}^{-2}$. In the case of QWrs, a periodic corrugation of the epilayer is obtained giving place to an array of wires aligned along the [1-10] direction with a typical length above $5 \mu \mathrm{m}$, a height between 0.6 and $2 \mathrm{~nm}$ and a period of $20 \mathrm{~nm}$. The samples have been characterized by AFM, TEM and photoluminescence. Growth and characterization details of InAs/InP samples have been published elsewhere (Gonzalez, 2000).

The DAFS measurements have been carried out at the french CRG (Colaborative Research Group) beamline BM02 at the ESRF (European Synchrotron Radiation Facility). Silicon (111) single crystals were used for beam monochromatisation. We performed the DAFS measurements at the As K-edge $(11867 \mathrm{eV})$ with an energy resolution better than $1 \mathrm{eV}$. We used Silicon photodiodes in photovoltaic mode for monitoring the incident beam and a $\mathrm{NaI}$ scintillator to record the diffracted intensity. The diffraction measurements were performed in glancing angle geometry, with an incidence angle close to the critical angle of InP and GaAs $\left(\sim 0.2^{\circ}\right)$ and kept constant during the scan. The critical angle was determined by scanning the intensity of the substrate Bragg peak as a function of the incidence angle.

The DAFS spectra were recorded by measuring the maximum intensity of the Bragg reflection as a function of energy (topDAFS-scan). The X-ray polarization vector was nearly perpendicular to the sample surface. We also recorded the glancing-angle EXAFS spectrum of the QWrs sample at the As Kedge, in fluorescence mode. The fluorescence yield was detected by means of a solid-state Ge detector.

\section{Results and discussion}

The XRD - $\Theta 2 \Theta$ spectrum of the QWrs sample, recorded at 11.8 $\mathrm{KeV}$, for the (440) Bragg reflection, is shown in Fig.2. The momentum transfer vector $\mathrm{q}$ is oriented along the [110] direction, i.e. perpendicular to the wires. The sharp Bragg peak at $\mathrm{q}$ about $0.96 \AA^{-1}$, corresponds to the (440) Bragg reflection from the InP 
substrate. Several satellites peaks due to the wires periodicity clearly appears close to the substrate peak. The satellites spacing gives a wire periodicity, $\Lambda=1 / \Delta \mathrm{q} \cong 200 \AA$, in fair agreement with the AFM image. The epilayer looks slightly relaxed in the direction perpendicular to the wires ([110]). Simulations are in progress to establish the exact matching degree between substrate and epilayer.

The (420) peak was also recorded and it showed a shape very similar to (440).

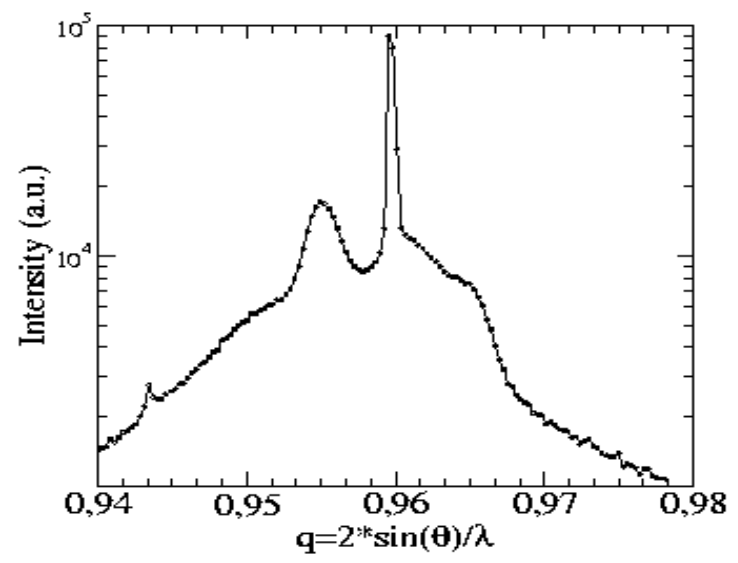

Figure 2. XRD intensity of QWrs sample, as a function of the momentum transfer, around the (440) InP Bragg reflection. The spectrum has been recorded in glancing-angle geometry at $11.8 \mathrm{KeV}$ (below As K-edge).

The XRD - $\Theta 2 \Theta$ spectrum of the QDs sample, for the (440) Bragg reflection, recorded at $11.87 \mathrm{KeV}$, is shown in Fig.3.

The broad peak, that shows at the base of the sharp substrate

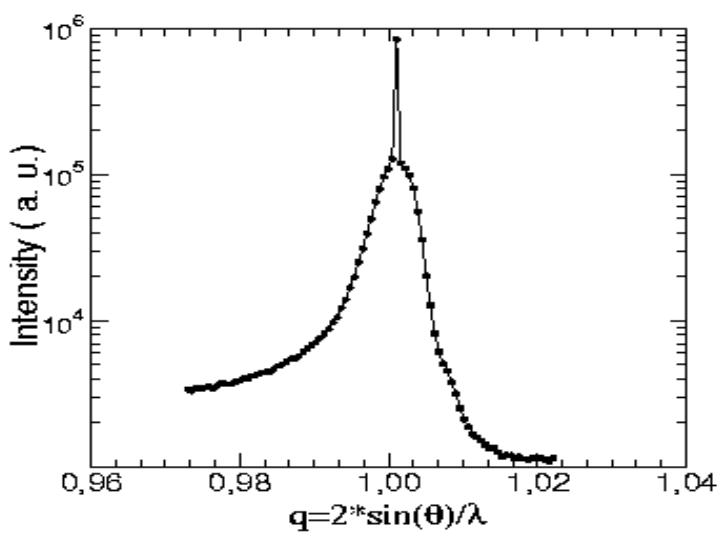

Figure 3. XRD patterns of (440) GaAs Bragg reflection, as a function of the momentum transfer, from QDs. The spectrum has been recorded in glancing-angle geometry at $11.87 \mathrm{KeV}$ (above As K-edge).

peak, is the dots contribution to the diffracted intensity. Its width is in agreement with an average Dots diameter of about $200 \AA$, as determined by AFM. Top-DAFS measurements were performed on the most intense satellite of the QWrs spectrum and on the high q-value side of the epilayer broad peak.

The DAFS spectra of the QWrs sample for (440) and (420) Bragg reflections are shown in Fig.4. A simultaneous crystallographic fit has been performed on the two spectra starting from the InAs bulk structure and allowing $\mathrm{P}$ atoms to substitute As in the epilayer.
The diffracted intensity was corrected for a transmission coefficient, $\left|\mathrm{T}_{\mathrm{yi}}\right|^{2}\left|\mathrm{~T}_{\mathrm{yf}}\right|^{2}$, that represents the effect of refraction, at the substrate surface, of the incoming X-ray wave. This correction has to be taken into account since, due to the low incidence angles, we get the total reflection regime and an evanescent $\mathrm{x}$-ray wave is generated beneath the surface, penetrating just a few nanometers into the refracting medium.

We applied the Distorted Born Wave Approximation (DBWA) (see for ex: Dosch, 1992), considering that total reflection takes place essentially at the substrate surface, i.e. considering the thin epilayer as a small perturbation, in terms of reflection and refraction, with respect to the flat bulk substrate.

The crystallographic fits are shown in fig. 4 as continuous curves. They were performed by using the DPU code (Wolfers). The fit parameters were the scale and slope factors to take into account geometrical and detection effects, Debye Waller factors and $\mathrm{P}$ concentration, $x$, for $\operatorname{InAs} s_{1-x} P_{x}$,

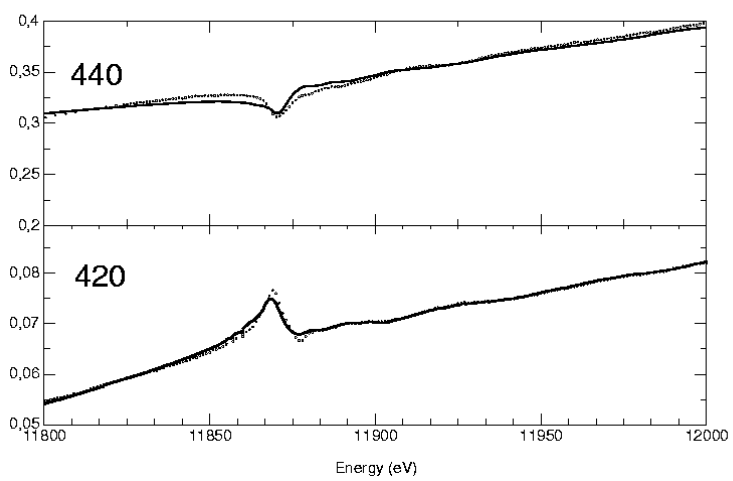

Figure 4. Glancing-angle DAFS spectra of QWrs, for (440) and (420) Bragg peaks, at the As K-edge.

The agreement between theory and experiment is quite good for (420) DAFS while less satisfactory for (440) spectrum. Further measurements are in progress to improve the data quality.

Nevertheless, as a preliminary, but clear result from the crystallographic fit, we have learned that to reproduce the shape of the anomaly at the edge, we do need to include the presence of $\mathrm{P}$ in the epilayer. For the QWrs sample we obtain $\mathrm{x} \cong 0.4+/-0.1$. This shows that a considerable amount of $\mathrm{P}$ atoms is incorporated in the wires. A strong intermixing of $\mathrm{P}$ and As is indeed expected at the interface buffer-layer/epilayer since the buffer surface is known to be P-rich. Therefore the first ML can contain a large P amount. The information that DAFS adds, is that this interface $\mathrm{ML}$ is already corrugated, i.e. makes part of the wires, otherwise it could not contribute to the satellites diffraction peaks due to the array periodicity.

The glancing-angle DAFS oscillations, after background subtraction, are shown in Fig.5 (dotted curve), together with the results of the crystallographic fit, obtained from the experimental $\mathrm{f}^{\prime}$ and $\mathrm{f}^{\prime}$ of bulk InAs (continuous curve). We did not perform any EXAFS fit since the quality of the spectrum has to be improved to detect the tiny changes in interatomic distances due to strain $(\sim 0.02 \AA)$ and to allow a next nearest neighbor analysis to detect the presence of $\mathrm{P}$ at the As K-edge.

Glancing-Angle EXAFS was also measured, on the same sample, detecting the As fluorescence yield. We report the EXAFS spectrum together with its Fourier Transform (FT) in Fig. 6. They are compared with the theoretical bulk InAs signals, calculated with the FEFF8 code (Rehr, 1991), and iterated, by means of FEFFIT program (Stern, 1995) to fit the experimental FT InAs contribution, represented by the peak at $2.5 \AA$. The best fit value 


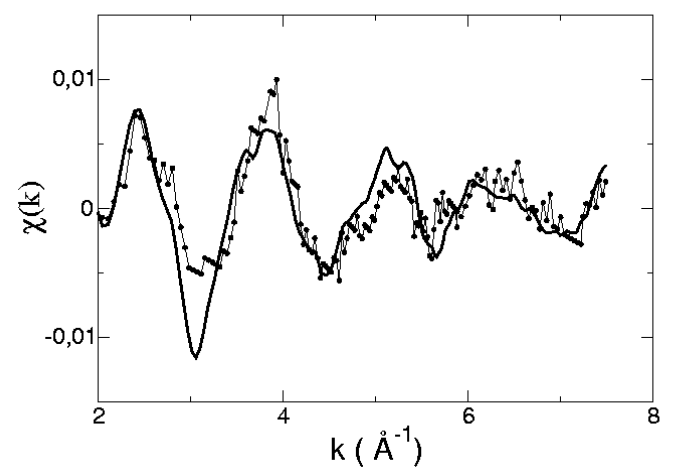

Figure 5: Glancing-angle EDAFS oscillations, at the As K-edge, of the InAs/InP QWrs array (dotted curve) after background subtraction, compared with the EDAFS on bulk InAs (continuous curve).

for the As-In distance was of $2.60 \pm 0.03 \AA$, i.e. equal within the error, to the bulk InAs value. The comparison with Fig. 5 is quite interesting: while EDAFS compares quite well with the correspondent DAFS spectrum of bulk InAs, the EXAFS signal is quite different from bulk InAs. It shows a clear As oxyde shape with a strong low-frequency component. It corresponds in the FT spectrum, to a huge peak at $1.2 \AA$. An analogous effect has been already observed for strongly oxydised GaAs surfaces (Proietti, 1993).

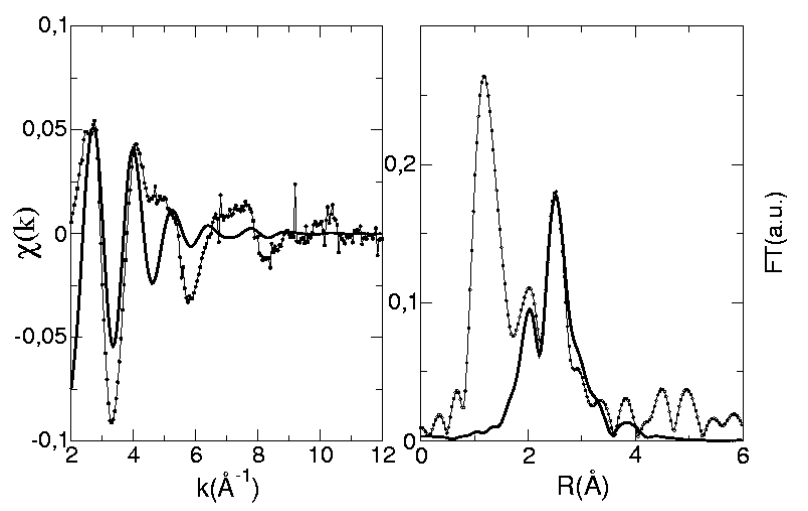

Figure 6. Glancing angle EXAFS and Fourier Transform, at the As Kedge, of the same sample as in Fig.5 (dotted curves), compared with InAs best fit signal from FEFF8.

It is interesting to note that the presence of an oxyde « dead» layer affects in a different way EXAFS and EDAFS. In the case of EDAFS it lowers mostly the overall diffracted intensity and the jump at the edge. A correction has been introduced in the DPU fit, (see for ex.: Dosch, 1992), depending on the dead layer thickness, which was then allowed to vary as a fit parameter. The oxyde thickness found was of $15+/-5 \AA$. On the other side, the EDAFS oscillations are not affected by the oxyde, due to the site selectivity of the diffraction structure factor. EXAFS instead, collects and averages out all the different As sites contributions, including the As-O numerous pairs.
The Glancing-Angle DAFS of the QDs sample, for the (440) Bragg reflection, is shown in Fig. 7. The crystallographic fit is made in this case more complicated by the presence of As in the substrate. All the corrections factors are now anomalous and strongly affect either the anomaly lineshape and the EDAFS oscillations. Further measurements are in progress to improve the signal quality and to allow a more reliable data analysis. Nevertheless, as it occurs for the QWrs sample, we cannot obtain any reasonable fit with a pure InAs epilayer.

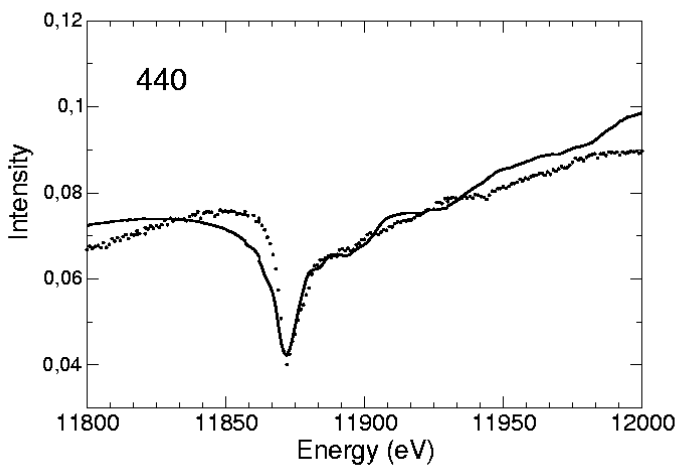

Figure 7. Glancing-angle DAFS spectra of QDs, for (440) Bragg reflection, at the As K-edge.

An analogous fit procedure shows the presence of $\mathrm{Ga}$ atoms substituting In in the QDs. The Ga concentration, $x$, obtained for $\mathrm{In}_{1-\mathrm{x}} \mathrm{Ga}_{\mathrm{x}} \mathrm{As}_{\mathrm{s}}$ is $\mathrm{x}=0.25+/-0.1$, showing, also for this system, a strong intermixing at the substrate interface. This has been recently observed on Ge/Si QDs (Boscherini, 2000), and an explanation has been suggested by those authors in terms of strain-enhanced diffusion to reduce the strain energy content of the islands.

We acknowledge the CRG-D2AM beamline for granting beamtime and technical assistance. This work was supported by the LEA-MANES European Agreement and by CICYT project No. MAT99-0847.

\section{References}

Boscherini, F., Capellini, G., Di Gaspare, L., Motta, N., Rosei, F., Mobilio, S., (2000) Appl. Phys. Lett. 76, 682-684.

Dosch, H., «Critical Phenomena at Surfaces and Interfaces », (1992)

126, Springer Tracts in Modern Physics, Springer Verlag.

Gonzalez, L., Garcia, J.M., Garcia, R., Martinez-Pastor, J., Ballesteros, C, (2000) Appl. Phys. Lett., 1104-1106.

Xie, Q., Madhukar, A., Chen, P. \& Kobayashi, N.P., (1995). Phys. Rev. Lett. 75, 2542-2545

Proietti, M.G., Garcia, J., Chaboy, J., Morier-Genoud, F. \& Martin, D.(1993). J. Phys. Condens. Matter 5, 1229-1235.

Rehr, J.J., Mustre de Leon, J., Zabinsky, S.I. \& Albers, R.C., (1991) J. Am. Chemm. Soc. 113, 5135-5145.

Stern, E.A., Newville, M., Ravel, B., Yacoby, Y. \& Haskel, D. (1995) Physica B 208\&209, 117-127.

Wolfers P. (Laboratoire de Cristallographie, private communication) 